\section{Searching for certainty}

\section{J. C. R. Hunt}

The Weather Revolution: Innovations and Imminent Breakthroughs in Accurate Forecasting. By Jack Fishman and Robert Kalish. Plenum: 1994. Pp. 276. \$27.95. The Scariest Place on Earth: Eye to Eye with Hurricanes. By David E. Fisher. Random House: 1994. Pp. 250. \$23.

WHEN even scientific journalists ask whether improvements in numerical weather prediction rely on empirical fixes, it is clearly high time that some good books should be written to explain to a wide audience the basis of recent progress in this great international endeavour. These two popular accounts go some way to meet this need. Both are written in journalistic prose, illustrated with a few useful line diagrams, and both provide informal histories of the scientific and technical discoveries that have led to modern meteorology and weather forecasting. Jack Fishman and Robert Kalish cover most aspects of weather forecasting and touch on aspects of global climate change, seasonal forecasting and the depletion (or 'hole') of ozone in the stratosphere. David E. Fisher, on the other hand, aims to cover a narrower theme in more depth, writing a story around his own experiences and those of other eye witnesses of Hurricane Andrew's impact on Miami in August 1992. The style of the books is reminiscent of O. G. Sutton's Understanding Weather (1960) or, more recently, James Gleick's Chaos (1989). But they contain hardly any discussion of the underlying mathematical ideas that are as relevant as the science and technology.

Fishman and Kalish nicely tell how modern weather forecasting has developed from empirical rules (whose 'unscientific' nature led the Royal Society to suppress forecasts in the United Kingdom from 1866 to 1877), to the application of physical principles (Buys Ballot's 'law' and the Bergen school's 'fronts' and 'airmasses') and then to the use of extensive computations, stemming from L. F. Richardson's visionary scheme of 1922 , which became practical only with the arrival of von Neumann's ENIAC electronic computer at Princeton in 1945, running at about $10^{2}$ floating point operations per second (FLOPS). Today's computer speeds are about $10^{10}$ FLOPS, with data from measurements ingested at about $10^{8}$ bits per day. Both these rates are rising exponentially by roughly a factor of between 10 and 100 every 10 years.

Fishman and Kalish rightly emphasize the enormous increase in data that have become available for improving weather forecasts from new kinds of 'remote sensing' measurements and images obtained from weather radars and satellites. But they might also have emphasized that, despite the enormous investment in these systems, their data are only now beginning to be effectively used for numerical predictions over longer periods, as a result of 'data assimilation' techniques originating from mathematical control theory. This is the first of the really new elements in the weather-forecasting revolution of the 1990s.

The second new element is the marked improvement in modelling the small-scale but vitally important features of atmospheric motion that cannot be calculated explicitly in the Richardson-like models of the large-scale motions. In a sense, the small-scale models are based on Luke Howard's remarkable insight in 1802 (which long pre-dated modern discussion of 'order from chaos') that there are only a few important types (or, in the language of physics, 'eigenstates') of atmospheric motion on this scale, which are made visible in the form of cloud patterns (stratus, cumulus and so on) - a point mentioned but not emphasized by Fishman and Kalish.

Hurricanes are essentially another eigenstate of atmospheric motion: once triggered, they develop a characteristic structure that persists for many days. This means that a generic subgrid scale model, together with satellite-image data, may be used to calculate hurricane tracks. The main numerical weather-forecasting centres are making more rapid progress than suggested in these books (see $\mathrm{New}$
Scientist, 28 January 1995, page 18).

Fisher describes the awesome power of hurricanes and the history of recent ones in the southeast Atlantic, some of which made landfall with devastating effect whereas others turned back or failed to develop. The physical principles of these vagaries are well covered.

Fishman and Kalish's last chapter, entitled "The revolution has begun", is an upbeat account of modern forecasting, especially at the 2-to-5-day period. They neglect to mention, however, that beyond this limited period it is impossible to make accurate deterministic forecasts, as $\mathrm{E}$. Lorenz demonstrated in 1963, thus negating Richardson's 1922 prophecy of numerical prediction becoming as accurate as the nautical almanac. The authors unwisely remark that later mathematical discoveries will perhaps enable Lorenz's theoretical limitation to be overcome - I

Eye of the storm - satellite image of Hurricane Andrew.

wouldn't bank on it. In fact, the third new element in weather forecasting in the 1990 s is the constructive recognition of this limit, so that forecasting is becoming more probabilistic, especially beyond about 5 days.

The weather interests everyone in different ways - as an exciting phenomenon to be described and classified, as a huge technical challenge involving measurements, forecasts and so on, or as a manifestation of many physical processes with complex mathematical behaviour, which overall are in a delicate state of dynamic, chemical and even biological balance. These authors have succeeded in popularizing the first two aspects. But I think they underplay and slightly misrepresent the theoretical concepts that are now guiding the development of modern meteorology, even in its most practical aspects.

J. C. R. Hunt is in the Meteorological Office, London Road, Bracknell, Berkshire RG12 2SZ, UK. 